\title{
Mitochondria Biogenesis Modulates Iron-Sulfur Cluster Synthesis to Increase Cellular Iron Uptake
}

\author{
Ping La, ${ }^{1, *}$ Joseph H. Oved, ${ }^{1,2, *}$ Valentina Ghiaccio, ${ }^{1}$ and Stefano Rivella ${ }^{1,3,4}$
}

Iron-sulfur (Fe-S) clusters are required for mitochondrial function. Fe-S cluster synthesis occurs in the mitochondria and iron uptake is required for mitochondrial biogenesis. However, $\mathrm{Fe}-\mathrm{S}$ clusters inhibit the expression of the iron importer transferrin receptor 1 (TfR1), whereas lack of the Fe-S cluster stimulates TfR1 expression. Yet, it is unclear whether Fe-S cluster synthesis increases with mitochondria biogenesis and, in turn, whether this negatively modulates TfR1 expression. We manipulated peroxisome proliferator-activated receptor-gamma coactivator-1 $\alpha$ expression to control mitochondrial biogenesis in a variety of cell types, including erythroid cells. We demonstrated that Fe-S cluster synthesis increases with mitochondria biogenesis but does not interfere with increasing TfR1 expression. In fact, TfR1 expression is stimulated through alternative means to meet iron requirement for mitochondria biogenesis. Furthermore, under enhanced mitochondria biogenesis, increased Fe$\mathrm{S}$ cluster synthesis inhibits the function of iron-regulating protein (IRP)1 and hence stimulates the expression of $5^{\prime}$-aminolevulinate synthase 2 (ALAS2), a target of IRP1 and rate-limiting enzyme in erythroid heme biogenesis. Increased ALAS2 expression leads to enhanced heme production, hemoglobinization, and erythropoiesis. Therefore, our study also provides a mechanism to link mitochondrial biogenesis with erythropoiesis and has a potential therapeutic value in the treatment of blood disorders.

Keywords: mitochondria biogenesis, iron-sulfur cluster, heme, erythropoiesis, TfR1

\section{Introduction}

I RON-SULFUR (FE-S) CLUSTERS are essential cofactors for mitochondrial functions and are also synthesized within the mitochondria (Rouault and Maio, 2017). Fe-S clusters inhibit the expression of the iron importer transferrin receptor 1 (TfR1), which blocks the iron uptake required for mitochondria biogenesis (Ishii et al., 2009; Rensvold et al., 2013; Zhang et al., 2014). It is unclear, however, whether $\mathrm{Fe}-\mathrm{S}$ cluster synthesis increases with mitochondria biogenesis and, in turn, whether this negatively modulates TfR 1 expression and thus interferes with mitochondrial iron demand.

Fe-S clusters are synthesized in the mitochondria and cytosol by two different Fe-S cluster assembly machineries (Rouault, 2015; Braymer and Lill, 2017). However, the early steps of cytosolic Fe-S cluster synthesis require the mitochondrial Fe-S cluster assembly machinery. This process is mediated by the mitochondrial transporter ABCB7 (Pondarre et al., 2006). Therefore, the mitochondrial assembly machinery is essential for all de novo Fe-S cluster synthesis.
Two RNA-binding proteins iron-regulating protein (IRP) 1 and IRP2 regulate iron metabolism by binding ironresponsive element (IRE) motifs. For instance, IRPs bind to the 3'UTR IREs of TfR1 mRNA, thereby stabilizing it and increasing iron import (Caspary et al., 1977). In contrast, IRPs binding to the 5'UTR IRE of 5'-aminolevulinate synthase 2 (ALAS2), the rate-limiting enzyme in the erythroid heme synthesis pathway, abrogate ALAS2 translation and iron consumption (Duncan et al., 1999). Iron and $\mathrm{Fe}-\mathrm{S}$ cluster levels regulate IRE-binding activity of IRPs and thereby affect iron homeostasis. Increasing levels of iron trigger IRP2 degradation by an ubiquitination mediated process (Salahudeen et al., 2009; Vashisht et al., 2009). In addition, Fe-S clusters can associate with IRP1 and convert it into cytosolic aconitase (aka ACO1), while simultaneously losing the IRE-binding activity (Haile et al., 1992). Therefore, increased iron and Fe-S cluster levels decrease IRE-binding activity of IRPs. This decreases iron intake by destabilizing TfR1 mRNA while increasing iron consumption by derepressing ALAS2 translation. In contrast,

\footnotetext{
${ }^{1}$ Division of Hematology, Department of Pediatrics, Children's Hospital of Philadelphia, University of Pennsylvania, Philadelphia, Pennsylvania, USA.

${ }^{2}$ Cell Therapy and Transplant Section, Division of Oncology, Department of Pediatrics, Children's Hospital of Philadelphia, Philadelphia, Pennsylvania, USA.

${ }^{3}$ Cell and Molecular Biology Affinity Group (CAMB)—Children's Hospital of Philadelphia, Philadelphia, Pennsylvania, USA.

${ }^{4}$ Penn Center for Musculoskeletal Disorders, University of Pennsylvania, Philadelphia, Pennsylvania, USA.

*These authors contributed equally to this study.
} 
decreased iron and Fe-S clusters levels enhance iron uptake and decrease iron consumption, ultimately maintaining iron homeostasis.

$\mathrm{Fe}-\mathrm{S}$ cluster synthesis modulates IRE-binding activity of IRP1, thereby orchestrating IRP1-targeted gene expression. Genetic mutations in genes that control Fe-S cluster synthesis cause $\mathrm{Fe}-\mathrm{S}$ cluster insufficiency, ultimately disturbing mitochondria function, inhibiting erythroid heme synthesis, and predisposing individuals to numerous diseases, including anemia and myelodysplastic syndromes (Ajioka et al., 2006; Pondarre et al., 2007; Ye et al., 2010; Bottomley and Fleming, 2014; Schmitz-Abe et al., 2015). Yet, it is unclear whether Fe-S cluster synthesis correlates with mitochondria biogenesis and whether this modulates iron uptake through changes on TfR1 expression. This is also relevant in erythropoiesis, a process that requires active mitochondrial biogenesis and consumption of the majority of physiological iron (Muckenthaler et al., 2017).

In this article, we show that Fe-S cluster synthesis correlates with mitochondria biogenesis without interfering with TfR1 expression and that this coordination in erythroid cells modulates ALAS2 expression and ultimately erythropoiesis.

\section{Materials and Methods}

\section{Cell lines}

Murine preadipocytes 3T3-L1 were cultured in Dulbecco's modified Eagle's medium containing $10 \%$ (v/v) fetal calf serum, penicillin (100 units $/ \mathrm{mL})$, and streptomycin $(100 \mu \mathrm{g} / \mathrm{mL})$, under $5 \% \mathrm{CO}_{2}$ and at $37^{\circ} \mathrm{C}$. Murine erythroleukemia (MEL) cells were cultured in Roswell Park Memorial Institute medium containing $10 \%$ (v/v) fetal calf serum, penicillin (100 units $/ \mathrm{mL})$, and streptomycin $(100 \mu \mathrm{g} / \mathrm{mL})$, under $5 \% \mathrm{CO}_{2}$ and at $37^{\circ} \mathrm{C}$. For differentiation, MEL cells were treated with $50 \mathrm{mM}$ hexamethylene bisacetamide (HMBA) for different days as indicated in figure legends.

\section{Western blot analysis}

Cells were lysed in radioimmunoprecipitation assay buffer with proteinase inhibitors. Protein concentrations were measured by using Pierce ${ }^{\mathrm{TM}}$ BCA Protein Assay Kit. The equal amount of total proteins was separated by sodium dodecyl sulfate-polyacrylamide gel electrophoresis and transferred to polyvinylidene difluoride (PVDF) membrane. The PVDF membrane was further incubated with different primary antibodies as indicated and followed by another incubation with horseradish peroxidase-conjugated secondary antibody to visualize the binding affinity of the primary antibody. Calnexin, GADPH, and Actin were used as an internal control.

\section{Quantitative-real time-PCR assay}

Total RNA was isolated by using RNeasy Mini kit (Qiagen), reverse transcribed into cDNAs by using SuperScript III Reverse Transcriptase (Thermo Fisher), and further analyzed by TaqMan Gene Expression Assays (Thermo Fisher). GAPDH and ACTB mRNA levels were used as an internal control.

\section{Aconitase activity assay}

In Gel assay was applied as described previously (Tong and Rouault, 2006; Ghosh et al., 2008). In brief, cell lysis was separated by PAGE with Tris-borate buffer and then incubated with the solution containing cis-aconitic acid, isocitric dehydrogenase, phenazine methyl sulfate, NADP, and 3-(4,5-dimethylthiazol-2-yl)-2,5-diphenyltetrazolium bromide at $37^{\circ} \mathrm{C}$ for $10 \mathrm{~min}$. After incubation, gels were photographed.

\section{Analysis of intracellular heme levels}

A colorimetric assay kit (Cat No: K672; Biovision Incorporated) was utilized per manufacturer's instructions. Three million cells were used for each sample. Experiments were done in duplicate, repeated at least three times each, and measured at OD570 using a 96-well plate reader.

\section{Mitochondria biogenesis assays}

Cells were labeled with $100 \mathrm{nM}$ Mitotracker Green FM or Deep Red FM (Thermo Fisher) and followed by fluorescence-activated cell sorting (FACS). FACS data were analyzed by using FlowJo7 software. In brief, cells in exponential growth phase were incubated with dye for $30 \mathrm{~min}$ at $37^{\circ} \mathrm{F}$ in culture medium. Cells were then collected and analyzed on a FACSCalibur (BD Biosciences). Data were collected from 10,000 cells and plotted as median fluorescent intensity.

\section{Analysis of IRE-binding activities}

IRE-binding activities were measured differently as shown in Figure 3. Figure 3 shows IRE-binding activity was analyzed as previously described (Meyron-Holtz et al., 2004). In brief, to generate IRE probe, IRE sequence derived from ferritin heavy chain mRNA was cloned and further labeled with phosphorus-32 radioactive isotope by using Riboprobe ${ }^{\circledR}$ Systems (Promega). Total protein was extracted from cells by using Triton X-100 buffer. As detailed previously, equal amount of total proteins was incubated with IRE probes and separated by PAGE followed by an exposure to X-ray films. As shown in Figure 5, IRE-binding activities were measured by using LightShift ${ }^{\mathrm{TM}}$ Chemiluminescent RNA EMSA Kit (Thermo Fisher) per manufacturer's instruction.

\section{Plasmid construction}

The Myc epitope was fused at the N-terminus of human peroxisome proliferator-activated receptor-gamma coactivator (PGC)- $1 \alpha$ cDNA and cloned into a retroviral vector (indicated as PMX-puro) carrying a puromycin resistant gene under control of the Moloney murine leukemia virus promoter and a lentiviral vector, indicated as EGW, which expresses the green fluorescent protein under control of the human elongation factor 1-alpha promoter. EGW, expressing the green fluorescent protein gene, was also utilized as a control. Retroviruses were used on 3T3-L1, whereas lentiviruses were used on MEL cells.

\section{Statistical analysis}

Each experiment was repeated for a minimum of three times. Data were analyzed by standard Student's $t$-test. Statistical significance was considered at $p<0.05$ versus corresponding controls or day 0 . 


\section{Results}

$P G C-1 \alpha$ induces Fe-S clusters synthesis

PGC- $1 \alpha$ is a transcriptional coactivator and plays a central role in governing mitochondrial biogenesis (Lin et al., 2005). To test whether Fe-S cluster synthesis increases with mitochondria biogenesis, human PGC-1 $\alpha$ tagged with an Myc epitope (Myc-PGC-1 $\alpha$ ) was expressed in preadipocytes 3T3-L1 (Fig. 1A). Expression was confirmed by Western blot. The Myc-PGC- $1 \alpha$ expression increased staining with Mitotrack Green and Deep Red (Fig. 1B), suggesting an increased mitochondria biogenesis. Moreover, Myc-PGC- $1 \alpha$ expression enhanced the gene expression of $5^{\prime}$-aminolevulinate synthase 1 (Fig. 1C), a rate-limiting enzyme in nonerythroid heme synthesis pathways (Handschin et al., 2005), and heme levels (Fig. 1D). Thus, these data confirmed that Myc-PGC- $1 \alpha$ functions similar to endogenous PGC- $1 \alpha$.
A

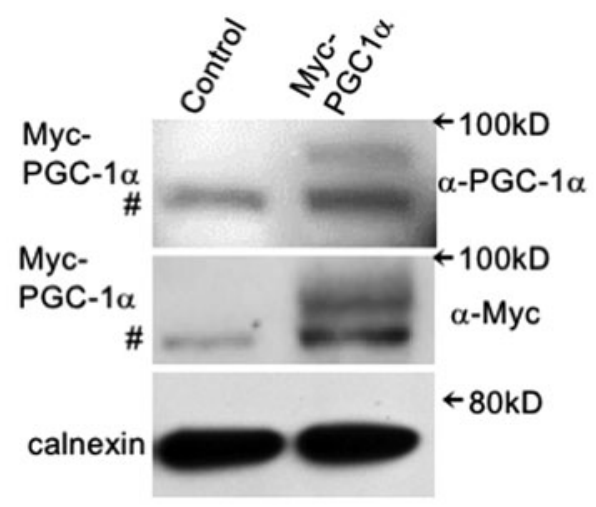

D

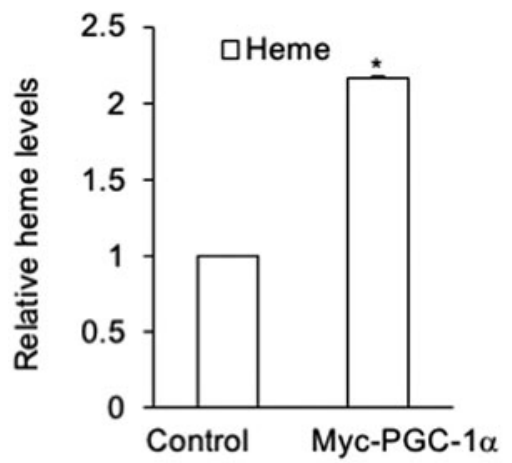

B

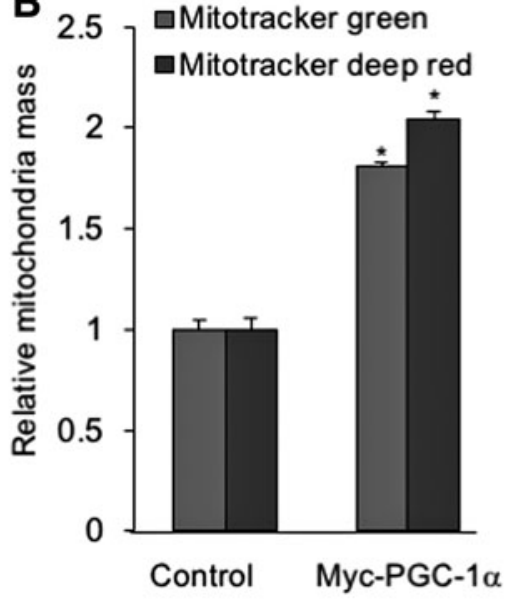

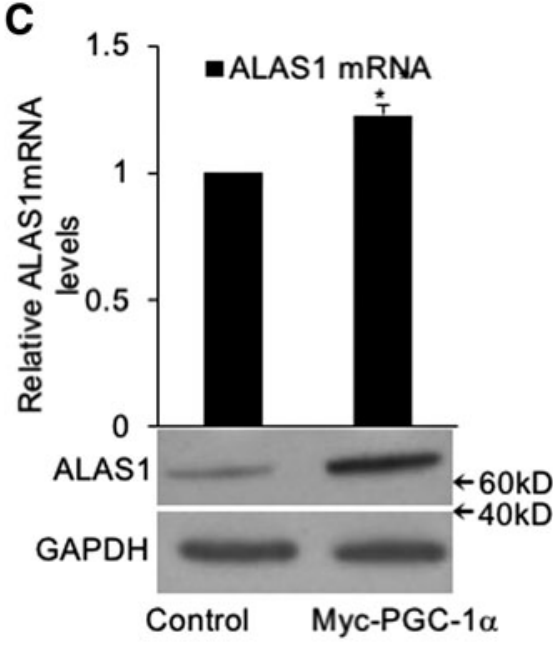

$\mathbf{F}$

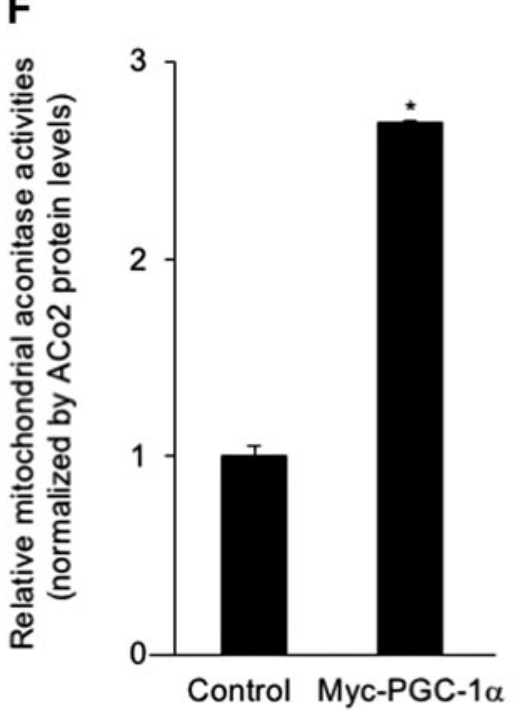

FIG. 1. PGC-1 $\alpha$ stimulates Fe-S cluster synthesis. 3T3-L1 cells were infected with retroviruses control or expressing Myc-PGC-1 $\alpha$ followed by puromycin selection. Puromycin-resistant cells were used for the following assays. (A) MycPGC-1 $\alpha$ expression was verified by using Western blot assay and anti-Myc and anti-PGC-1 $\alpha$ antibodies. (B) Mitochondria biogenesis was verified by Mitotrack Green and Deep Red staining and further FACS analysis. Mitotrack Green is proportional to mitochondrial mass, whereas Deep Red identifies alive mitochondria. (C) ALAS1 protein and mRNA levels were measured, respectively, by Western blot and qRT-PCR. ALAS2 mRNA levels were normalized by GAPDH mRNA amount. (D) Cellular heme contents were quantified by heme assay kit. (E) The activity and protein levels of aconitase were measured by In-Gel aconitase activity (top panel) and Western blot assays (lower panels). (F) Signals for the activity and protein levels of mitochondria aconitase (ACO2) shown in (D) were quantified by ImageJ software. The quantity produced from ACO2 aconitase activity was normalized by the number generated from ACO2 protein levels. Subsequently, this ratio was compared with that obtained from control cells. ${ }^{\#}$ A nonspecific band. Standard bars were generated by two replicates. Data were analyzed by standard Student's $t$-test. Statistical significance was considered at $p<0.05$ versus corresponding controls. These experiments were repeated more than three times. ${ }^{*} p<0.05$. ALAS1, $5^{\prime}$-aminolevulinate synthase 1 ; ALAS2, 5'-aminolevulinate synthase 2; FACS, fluorescence-activated cell sorting; Fe-S, iron-sulfur; PGC, peroxisome proliferator-activated receptor-gamma coactivator; qRT, quantitative-real time. 
To investigate whether mitochondria biogenesis affected Fe-S cluster synthesis, control and Myc-PGC-1 $\alpha$ expressing 3T3-L1 cells were analyzed for aconitase activities. Aconitases, represented by the cytosolic form $\mathrm{ACO} 1$ and mitochondrial form $\mathrm{ACO} 2$, require Fe-S cluster to be enzymatically active. Their activity was used to monitor the status of Fe-S cluster synthesis in cytosol (ACO1) and mitochondria (ACO2). By an in-Gel aconitase activity assay (Tong and Rouault, 2006), ACO1 and ACO2 proteins were separated by PAGE and then incubated with an aconitase substrate, resulting in a blue color whose intensity is proportional to aconitase activity. Myc-PGC- $1 \alpha$ expression significantly increased ACO1 activity despite limited increase in protein levels (Fig. 1E). This increased activity likely indicates enhanced Fe-S cluster synthesis (a known cofactor for ACO1) in the cytosol. For ACO2, Myc-PGC- $1 \alpha$ expression increased both protein levels and activity. However, simple overexpression of ACO2 protein in 3T3-L1 did not increase its activity (data not shown). Moreover, we quantified ACO2 activity (shown by the blue intensity in Fig. 1E, first panel from the top) and protein levels obtained by Western blot analysis (Fig. 1E, third panel from the top), then divided the former by the latter to normalize ACO2 activity by its protein levels. Compared with control cells, Myc-PGC- $1 \alpha$ overexpression increased ACO 2 activity by a factor of 2.7 (Fig. 1F), confirming that such an increase was due to an elevated $\mathrm{Fe}-\mathrm{S}$ cluster synthesis in mitochondria.

Since Fe-S cluster synthesis is stimulated under enhanced mitochondria biogenesis, we next investigated whether this stimulation was due to increased gene expression of the Fe-
S cluster assembly machinery. A subset of genes involved in this pathway was investigated. The protein levels of ISD11, ISCU, and FXN, which belong to the mitochondrial core assembly machinery, were increased by Myc-PGC- $1 \alpha$ expression (Fig. 2A). This was observed also for ISCA $1 / 2$ and Nfu1, which belong to the mitochondrial Fe-S cluster delivery machinery, and for ABCB7 that is involved in $\mathrm{Fe}-\mathrm{S}$ clusters mitochondrial export machinery and required for cytosolic Fe-S cluster synthesis (Rouault and Maio, 2017). For ISCU and Nfu1, the mRNA level did not change significantly and proportionally to their protein levels (Fig. 2B), suggesting a post-transcriptional regulation consistent with previous reports (Rensvold et al., 2013).

\section{Fe-S cluster synthesis is associated with mitochondria biogenesis but does not block mitochondria-required iron uptake}

As enhanced mitochondria biogenesis stimulates heme and $\mathrm{Fe}-\mathrm{S}$ cluster synthesis, two major iron-consuming pathways, we explored how TfR1 expression was regulated by mitochondria biogenesis-associated $\mathrm{Fe}-\mathrm{S}$ cluster synthesis. Since enhanced Fe-S cluster availability might decrease the IRE-binding activity of IRP1 (Zhang et al., 2014), we analyzed IRPs/IRE activity. As shown in Figure 3A, the IREbinding activity in Myc-PGC-1 $\alpha$-expressing 3T3-L1 cells was reduced $>50 \%$ compared with that in control cells, whereas IRP1 and IRP2 levels remained the same in control and Myc-PGC- $1 \alpha$-expressing cells. Along with reduced IRE-binding activity, TfR1 mRNA was decreased (Fig. 3B).

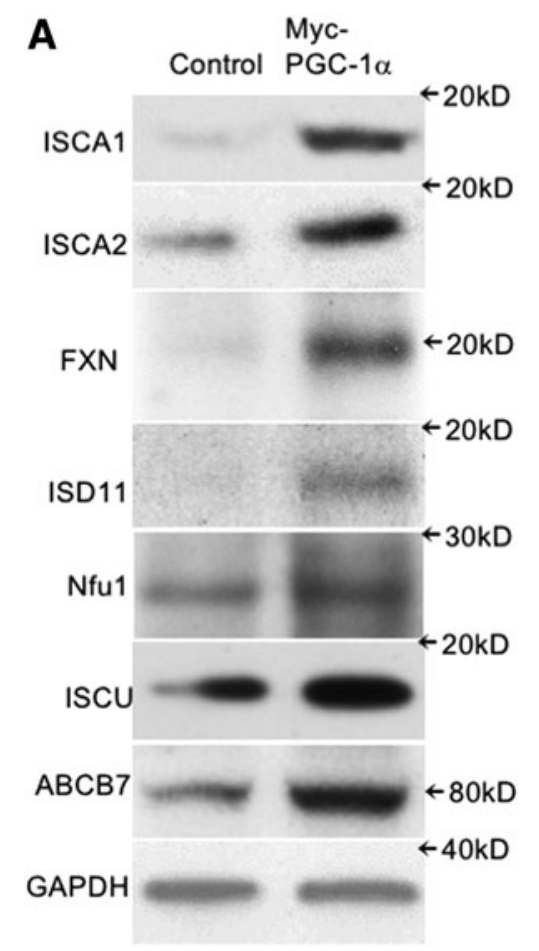

B

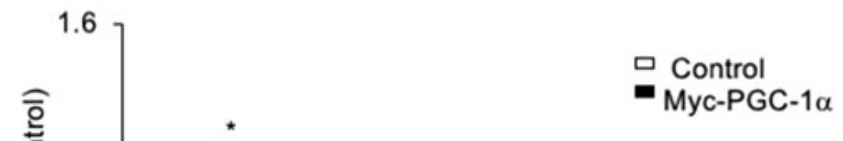

FIG. 2. PGC-1 $\alpha$ stimulates the gene expression of Fe-S cluster assembly machinery. (A) 3T3-L1 cells were infected with virus control or expressing Myc-PGC-1 $\alpha$ followed by puromycin selection. Puromycin-resistant cells were analyzed for protein levels of genes involved in Fe-S cluster assembly. (B) The same cells used in (A) were analyzed for the mRNA levels of Fe-S cluster assembly machinery, which were further normalized by GAPDH mRNA amount. Standard bars were generated by two replicates. Data were analyzed by standard Student's $t$-test. Statistical significance was considered at $p<0.05$ versus corresponding controls. These experiments were repeated more than three times. ${ }^{*} p<0.05$. 

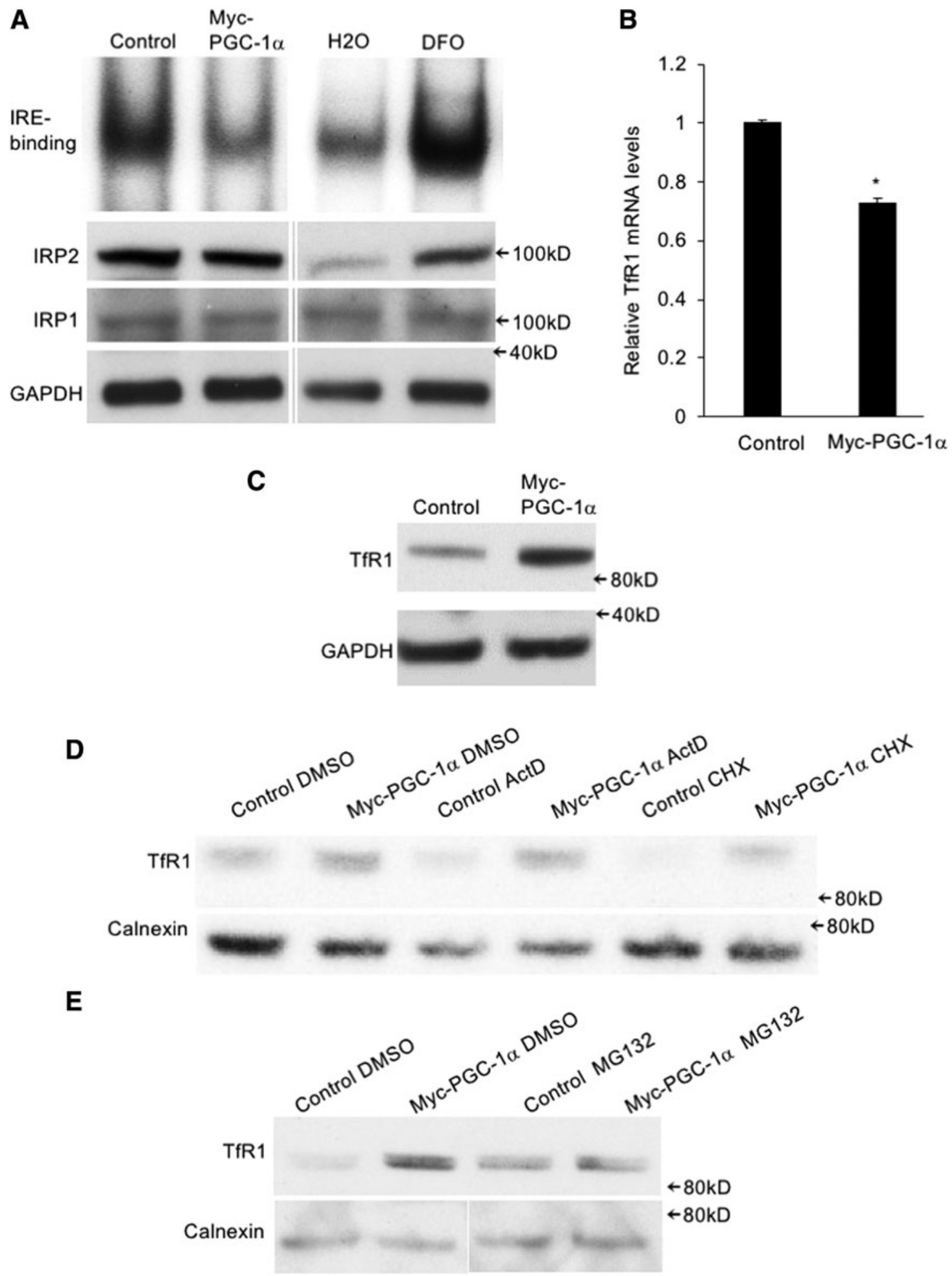

FIG. 3. Under enhanced mitochondria biogenesis in 3T3-L1 cells, Fe-S cluster synthesis is increased, whereas TfR1 protein levels are increased. (A) Control and Myc-PGC-1 $\alpha$-expressing 3T3-L1 cells were analyzed for IRE-binding activity (top panel) and IRPs levels (lower panels). The iron chelator DFO treatment was used as positive control, showing increased IRE-binding activity of IRPs due to IRP2 stabilization. (B) Control and Myc-PGC-1 $\alpha$-expressing 3T3-L1 cells were evaluated for TfR1 mRNA (B) and protein (C) levels. TfR1 mRNA levels were normalized by GAPDH mRNA amount. Standard bars were generated by two replicates. Data were analyzed by standard Student's $t$-test. Statistical significance was considered at $p<0.05$ versus corresponding controls. (D) Control and Myc-PGC-1 $\alpha$-expressing 3T3-L1 cells were treated with $0.05 \mu \mathrm{g} / \mathrm{mL}$ actinomycin $\mathrm{D}$ or cycloheximide for $24 \mathrm{~h}$ followed by Western blot assay. (E) Similar to (D), cells were treated with $0.1 \mu \mathrm{M} \mathrm{MG} 132$ for $32 \mathrm{~h}$ and analyzed. These experiments were repeated more than three times. $* p<0.05$. DFO, desferoxamine; IRE, iron-responsive element; IRP, iron-regulating protein; TfR1, transferrin receptor 1. 

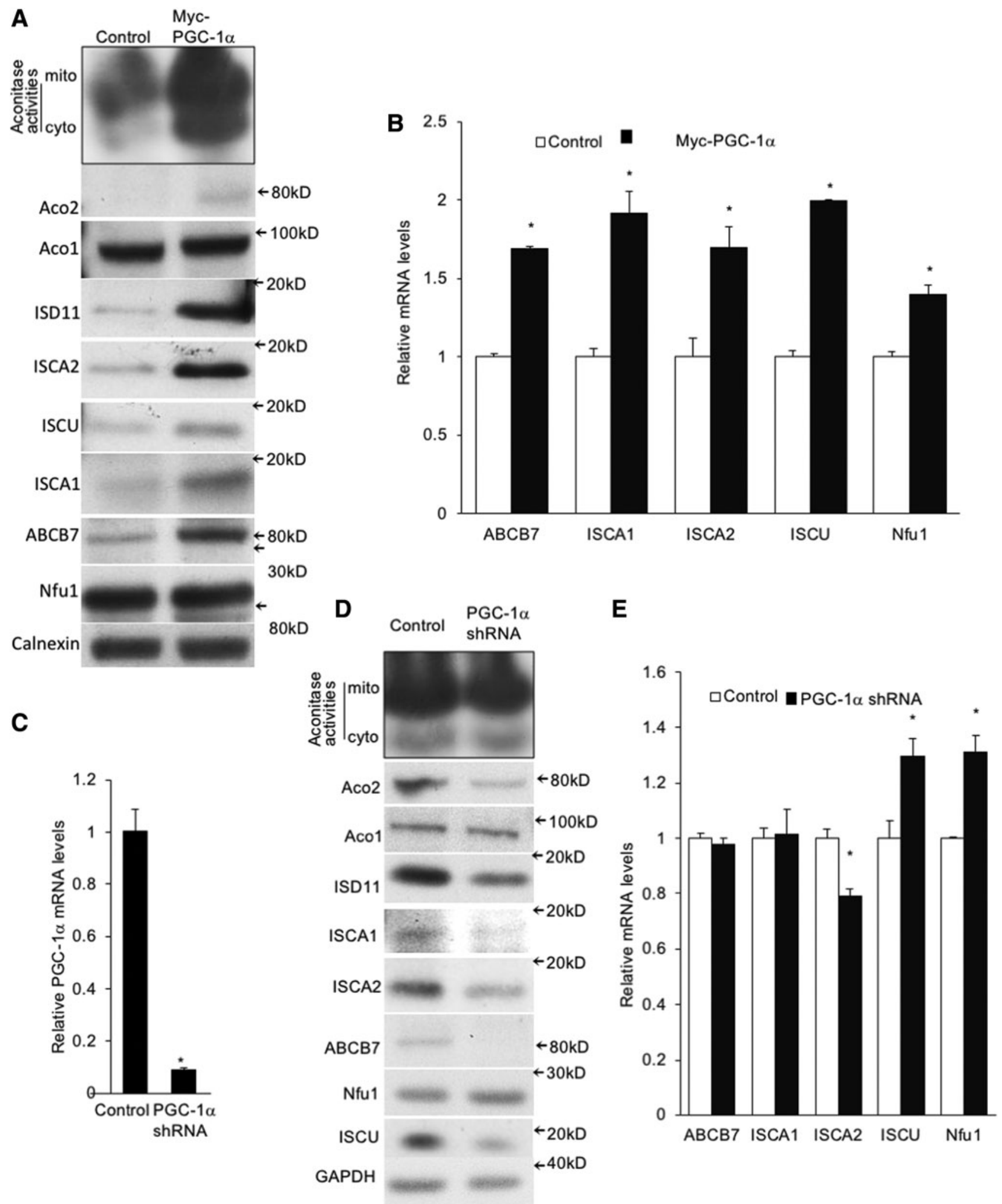

FIG. 4. Fe-S cluster assembly is associated with mitochondria biogenesis in erythroid cells. (A) MEL cells were infected with lentiviruses control or expressing Myc-PGC-1 $\alpha$. One day after infection, these cells were differentiated by incubation with $50 \mathrm{mM}$ HMBA for 5 days followed by the aconitase activity and Western blot assays. (B) Similarly, cells used in (A) were analyzed by qRT-PCR assays. (C) MEL cells were infected with lentiviruses shRNA control or targeting murine PGC-1 $\alpha$ mRNA and selected with puromycin. Puromycin-resistant cells were differentiated with $50 \mathrm{mM}$ HMBA incubation for 6 days for differentiation. Cells were analyzed for PGC- $1 \alpha$ mRNA levels by qRT-PCR assay. (D) Cells used in (C) were analyzed by Western blot analysis. (E) Similar to (C) and (D), the same cells were analyzed by qRT-PCR assay. Standard bars were generated by two replicates. Data were analyzed by standard Student's $t$-test. Statistical significance was considered at $p<0.05$ versus corresponding controls. These experiments were repeated more than three times. The mRNA levels for individual genes were normalized by ACTB mRNA amount. $* p<0.05$. HMBA, hexamethylene bisacetamide; MEL, murine erythroleukemia. 
However, in Myc-PGC-1 $\alpha$-expressing 3T3-L1 cells, TfR1 protein levels were increased, suggesting enhanced iron intake (Fig. 3C). To better understand TfR1 regulation, MycPGC-1 $\alpha$-expressing cells were incubated with either a proteasome inhibitor (MG132), a transcription inhibitor (actinomycin D), or a translation inhibitor (cycloheximide).
Incubation with MG132 reversed the increased TfR1 protein levels, whereas actinomycin D and cycloheximide had limited effect (Fig. 3D, E). These data suggest that increased TfR1 expression is primarily at the post-translational level and not at the transcription or translational level. Possibly, increased ATP production due to the enhanced
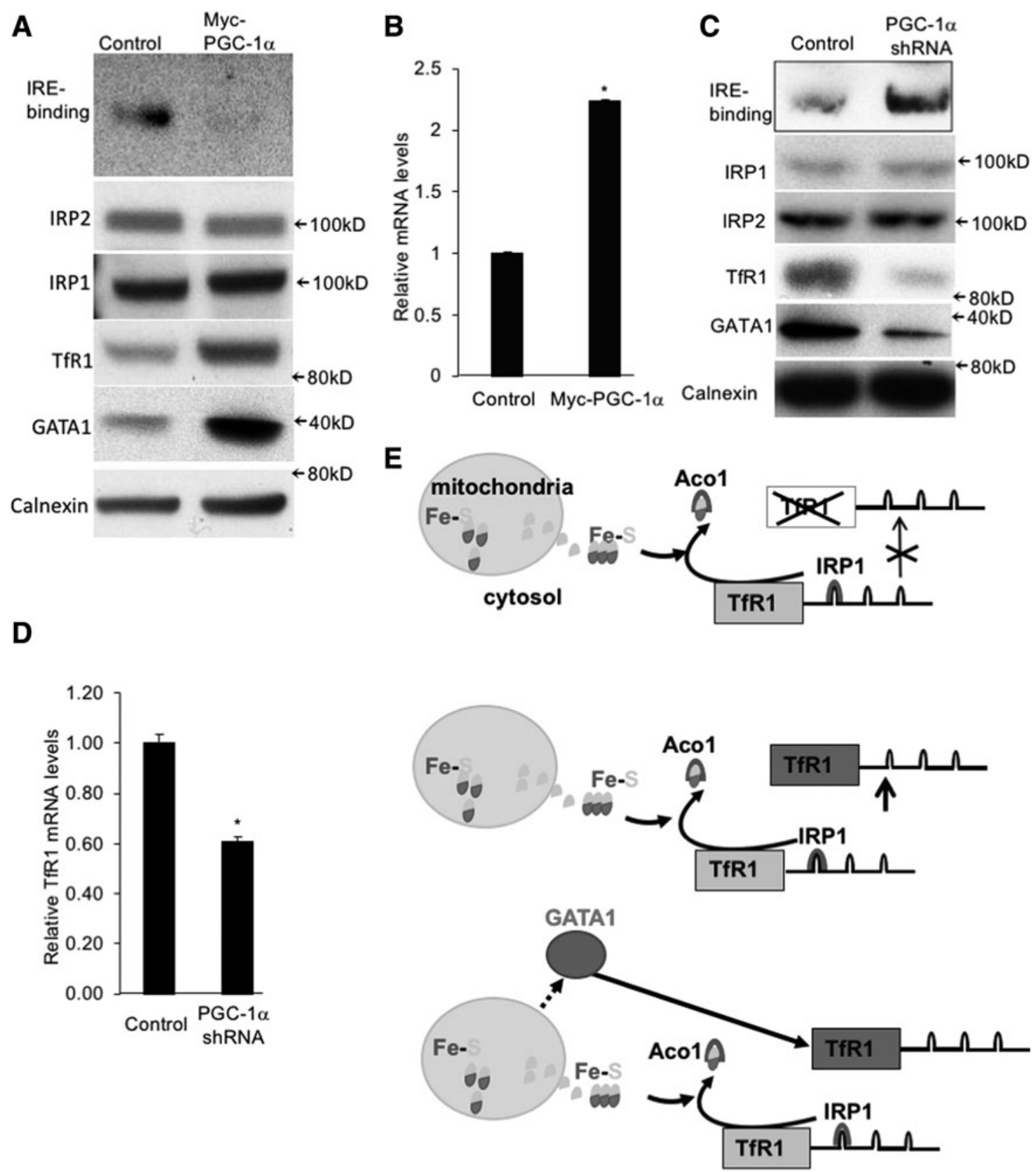

FIG. 5. In erythroid cells, there is differential GATA1 expression associated with mitochondria biogenesis and potentially regulates TfR1 gene expression. (A, B) MEL cells control or expressing Myc-PGC-1 $\alpha$ were differentiated and then analyzed by IRE-binding activity (A, the top panel) and Western blot assays (A, the lower panels) and for TfR1 mRNA levels by qRT-PCR (B). (C, D) Control and PGC-1 $\alpha$ shRNA-targeted MEL cells were generated by lentiviral infection and puromycin selection. After differentiation, these cells were analyzed by IRE-binding activity (the top panel) and Western blot assays (C) and for TfR1 mRNA levels by qRT-PCR (D). (E) The model for different regulations on TfR1 expression. Top: the canonical regulation mediated by Fe-S cluster synthesis; middle: the regulation mediated by increased IRP1 expression; bottom: the regulation mediated by GATA1. Standard bars were generated by two replicates. Data were analyzed by standard Student's $t$-test. Statistical significance was considered at $p<0.05$ versus corresponding controls. These experiments were repeated more than three times. The mRNA levels for individual genes were normalized by ACTB mRNA amount. $* p<0.05$. 
mitochondrial biogenesis potentiates TfR 1 folding and refolding to avoid degradation, although this requires further investigation. Nonetheless, these data demonstrate that Fe-S clusters have differential synthesis-based PGC- $1 \alpha$-mediated mitochondria biogenesis but does not limit mitochondriarequired iron uptake.

\section{In erythropoiesis, Fe-S cluster assembly is associated with mitochondria biogenesis but not iron uptake and ultimately modulates heme synthesis and hemoglobinization}

The regulation of iron metabolism, especially heme metabolism, is always different between erythroid and nonerythroid tissues (Ajioka et al., 2006; Muckenthaler et al., 2017). Therefore, we also investigated how mitochondria biogenesis, Fe-S cluster synthesis, and iron uptake maintain homeostasis during erythropoiesis by using murine erythroleukemia (MEL) cells, an in vitro model of erythropoiesis (Cui et al., 2014). First, we introduced Myc-PGC- $1 \alpha$ into MEL cells by viral transduction and differentiated these cells into erythrocytes by incubation with HMBA. As shown in Figure 4A and B, Myc-PGC- $1 \alpha$ expression stimulated the activity and expression of $\mathrm{Fe}-\mathrm{S}$ cluster assembly machinery, as evidenced by increased ACO1 and ACO2 activities as well as increased mRNA and protein levels of genes involved in Fe-S cluster synthesis. Next, we explored the impact of decreasing mitochondria biogenesis by lentiviral transduction of PGC- $1 \alpha$
shRNA (Fig. 4C). As shown in Figure 4D, protein levels of ISD11, ISCU, ABCB7, and ISCA1/2 were decreased in PGC$1 \alpha$-knockdown cells versus control. Subsequently, Fe-S cluster synthesis was also inhibited, as demonstrated by decreased aconitase activities of ACO1 and ACO2 (Fig. 4D top panel) and confirming a coordination between $\mathrm{Fe}-\mathrm{S}$ cluster synthesis and mitochondria biogenesis in erythroid cells. Similar to nonerythroid cells, for some genes of the assembly machinery, we did not observe the same trend between mRNA and protein level changes, especially when PGC- $1 \alpha$ expression was suppressed (Fig. 4E). Nonetheless, these data demonstrated that Fe-S clusters are also differentially synthesized based on mitochondria biogenesis in erythroid cells.

Next, we examined how mitochondrial biogenesis and Fe-S cluster synthesis affect iron demand, namely TfR1 expression in erythroid cells. As shown in Figure 5A, the IRE-binding activity was decreased in Myc-PGC- $1 \alpha$ expressing cells, whereas IRP levels remained similar. However, in contrast to this decreased IRE-binding activity, the mRNA and protein levels of TfR1 were both increased (Fig. 5A, B), suggesting an IRE-binding and IRP-independent mechanism. Indeed, we found that Myc-PGC- $1 \alpha$ stimulated the expression of GATA1 (Fig. 5A), a master regulator of erythropoiesis that has been shown to upregulate TfR1 expression (Kaneko et al., 2012). Corresponding to the changes in Myc-PGC-1 $\alpha$-expressing MEL cells, the opposite variations were demonstrated in PGC- $1 \alpha$-knockdown MEL cells, such as increased IRE-binding activity and

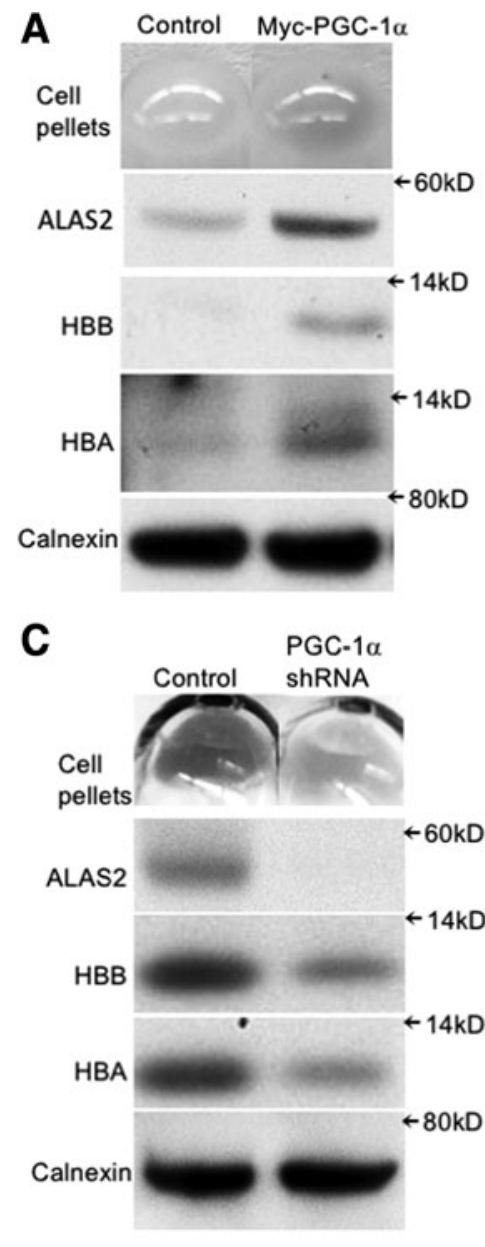

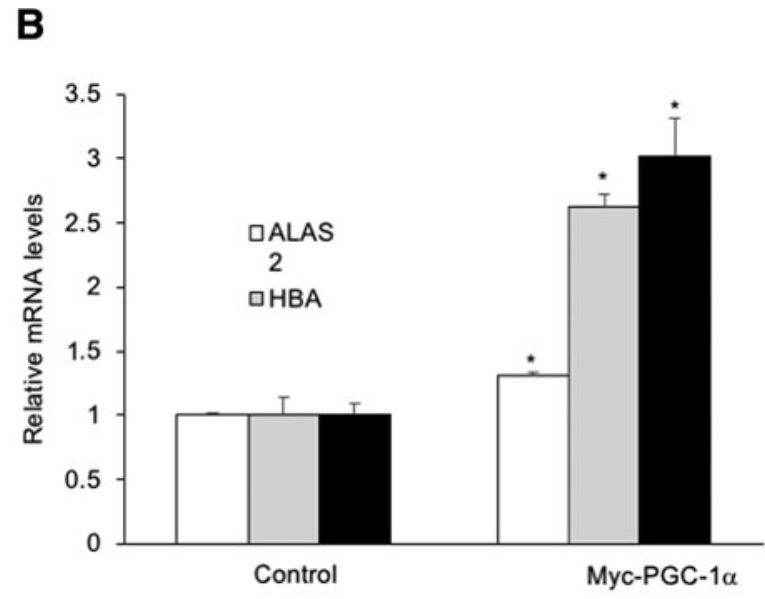

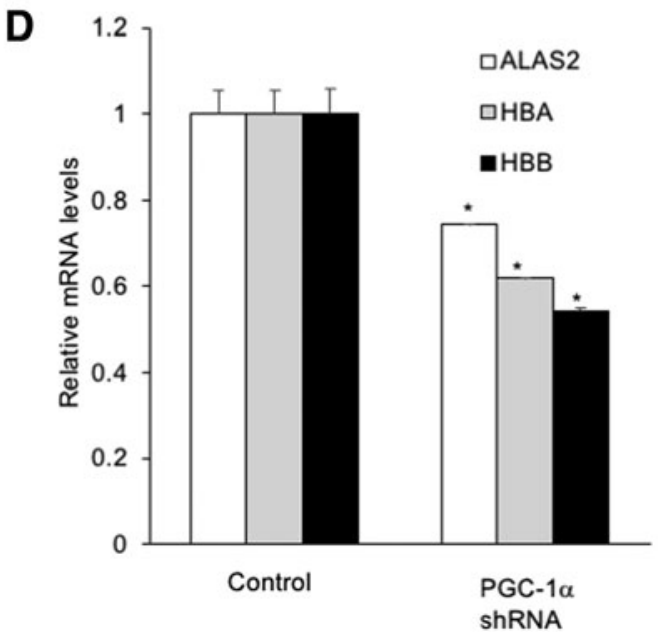

FIG. 6. Erythroid heme synthesis and hemoglobinization correlate with mitochondria biogenesis.

(A) Control or Myc-PGC- $1 \alpha$ expressing MEL cells were differentiated with HMBA incubation for 5 days, then pelleted and photographed (top panel of $\mathbf{A}$ ) followed by Western blot assays. (B) The same cells used in (A) were analyzed by qRT-PCR. (C) Control and PGC-1 $\alpha$ shRNA-targeted MEL cells were differentiated with HMBA treatment for 6 days, pelleted and photographed (top panel) followed by Western blot assay. (D) The same cells used in (C) were analyzed by qRT-PCR. The mRNA levels for individual genes were normalized by ACTB mRNA amount.

Standard bars were generated by two replicates. Data were analyzed by standard Student's $t$-test. Statistical significance was considered at $p<0.05$ versus corresponding controls. These experiments were repeated more than three times. ${ }^{*} p<0.05$. 
decreased TfR1 expression (Fig. 5C, D). Again, we observed a decreased GATA1 expression in PGC- $1 \alpha$ knockdown MEL cells, suggesting that unlike the mechanisms employed by 3T3-L1 (Fig. 5E, top and middle), erythroid GATA1 expression was upregulated under enhanced mitochondria biogenesis. This upregulation of GATA1 overrides the negative effect of increased $\mathrm{Fe}-\mathrm{S}$ cluster synthesis on TfR1 expression to meet the iron demand (Fig. 5E, bottom).

Next, we investigated how Fe-S cluster synthesis under enhanced mitochondria biogenesis regulates ALAS2 expression and potentially erythropoiesis. ALAS2 contains a 5'UTR IRE, and its translation can be inhibited by IRPs binding. Overexpression of Myc-PGC- $1 \alpha$ in MEL cells significantly stimulated ALAS2 levels and further hemoglobinization was evidenced by a more reddish color of cell pellets (Fig. 6A). As heme stimulates the expression of both $\alpha$-globin (HBA) and $\beta$-globin genes (HBB) (Tahara et al., 2004a, 2004b; Chen, 2014), we found that HBB and HBA expression was also stimulated. In contrast, PGC- $1 \alpha$ knockdown decreased the expression of ALAS2, HBB, and HBA as well as hemoglobinization (Fig. 6C, D). Of note, because PGC- $1 \alpha$ antagonizes the mitophagy that is required for the late stage of erythropoiesis (Lin et al., 2005; Grosso et al., 2017), Myc-PGC-1 $\alpha$-expressing cells were treated with HMBA for a short period of time, only 5 days to avoid this detrimental effect. Nonetheless, these data suggested that erythroid $\mathrm{Fe}-\mathrm{S}$ cluster synthesis is associated with mitochondria biogenesis without limiting iron uptake, but rather improving the synthesis of heme and hemoglobin.

\section{Discussion}

In this study, we demonstrated that Fe-S cluster synthesis is associated with mitochondria biogenesis but does not block mitochondria biogenesis-required TfR1 expression. In fact, TfR1 expression is stimulated through alternative means to meet iron requirement for increased mitochondria biogenesis. Furthermore, there is differential expression of ALAS2, HBB, and HBA that correlates with mitochondria biogenesis through $\mathrm{Fe}-\mathrm{S}$ cluster synthesis and transcription factor GATA1, thereby providing a mechanism connecting mitochondria biogenesis and erythropoiesis.

We showed that cytosolic Fe-S cluster synthesis is in tandem with mitochondria biogenesis. This could be due to the requirement of mitochondrial Fe-S cluster assembly machinery for cytosolic Fe-S cluster synthesis (Rouault and Maio, 2017). Interestingly, this allows the IRE-binding activity of IRP1, differential ALAS2 expression, and erythroid heme biosynthesis to be associated with changes in mitochondria biogenesis. We additionally demonstrated differential GATA1 expression associated with mitochondria biogenesis, further supporting the correlation between mitochondria biogenesis and ALAS2, HBA, and HBB gene expression. Moreover, heme level also promotes the transcription and translation of $\mathrm{HBB}$ and HBA through the transcription inhibitor bric-a-brac zinc finger domain and cap 'n'collar type of basic region leucine zipper factor homolog 1 (Bach1) and heme-regulated inhibitor, an EIF2A kinase (Tahara et al., 2004a, 2004b; Chen, 2014). Therefore, our study further demonstrates that erythropoiesis is associated with mitochondria biogenesis on both a transcription and post-transcription level and through Fe-S cluster synthesis as well as GATA1 signaling.
As demonstrated previously, TfR1 protein levels increase with mitochondria biogenesis (Ishii et al., 2009; O'Hagan et al., 2009; Rensvold et al., 2013). In this study, we demonstrate that despite increased Fe-S cluster synthesis, TfR1 expression was regulated with cell type-specific mechanisms to meet iron demand (Fig. 5E). For instance, 3T3-L1 cells post-translationally stabilized TfR 1 protein, possibly due to an increased mitochondrial ATP generation, which could facilitate TfR1 folding and refolding. In erythrocytes, GATA1 expression was increased under enhanced mitochondria biogenesis and could be responsible for stimulated TfR1 expression. However, it is unclear how GATA1 gene expression is regulated in line with mitochondria biogenesis. This question warrants future studies to identify mechanisms, which might be exploited to correct dysfunctional mitochondria and iron metabolism (Fleming, 2011; Ginzburg and Rivella, 2011; Chiang et al., 2016; Rouault, 2016). Given the wide-ranging downstream effectors of GATA1 activation, it will be critical to understand how maintenance of TfR1 expression potentially responds to GATA1 expression levels.

In conclusion, our data indicate that Fe-S cluster synthesis is associated with mitochondria biogenesis but does not limit mitochondria iron uptake. Ultimately, our study demonstrates that under enhanced mitochondria biogenesis, cells employ different routes to stimulate TfR 1 expression and ensure iron intake. It also suggests a mechanism underlying the essential role of mitochondria biogenesis in erythropoiesis. These data are important for devising new therapeutic modalities for a variety of dyserythropoietic diseases.

\section{Disclosure Statement}

S.R. is a consultant for Ionis Pharmaceuticals, Disc Medicine, MeiraGTx, and Protagonist Therapeutics.

\section{Funding Information}

This study was supported by the National Institutes of Health, the National Institute of Diabetes and Digestive, and Kidney Diseases grants R01 DK095112 and R01 DK090554 (S.R.)

\section{References}

Ajioka, R.S., Phillips, J.D., and Kushner, J.P. (2006). Biosynthesis of heme in mammals. Biochim Biophys Acta 1763, 723-736.

Bottomley, S.S., and Fleming, M.D. (2014). Sideroblastic anemia: diagnosis and management. Hematol Oncol Clin North Am 28, 653-670, v.

Braymer, J.J., and Lill, R. (2017). Iron-sulfur cluster biogenesis and trafficking in mitochondria. J Biol Chem 292, 12754-12763.

Caspary, W.F., Zavada, I., Reimold, W., Deuticke, U., Emrich, D., and Willms, B. (1977). Alteration of bile acid metabolism and vitamin-B12-absorption in diabetics on biguanides. Diabetologia 13, 187-193.

Chen, J.J. (2014). Translational control by heme-regulated eIF2alpha kinase during erythropoiesis. Curr Opin Hematol 21, 172-178.

Chiang, S., Kovacevic, Z., Sahni, S., Lane, D.J., Merlot, A.M., Kalinowski, D.S., et al. (2016). Frataxin and the molecular mechanism of mitochondrial iron-loading in Friedreich's ataxia. Clin Sci (Lond) 130, 853-870.

Cui, S., Tanabe, O., Lim, K.C., Xu, H.E., Zhou, X.E., Lin, J.D., et al. (2014). PGC-1 coactivator activity is required for murine erythropoiesis. Mol Cell Biol 34, 1956-1965. 
Duncan, R., Faggart, M.A., Roger, A.J., and Cornell, N.W. (1999). Phylogenetic analysis of the 5-aminolevulinate synthase gene. Mol Biol Evol 16, 383-396.

Fleming, M.D. (2011). Congenital sideroblastic anemias: iron and heme lost in mitochondrial translation. Hematology Am Soc Hematol Educ Program 2011, 525-531.

Ghosh, M.C., Tong, W.H., Zhang, D., Ollivierre-Wilson, H., Singh, A., Krishna, M.C., et al. (2008). Tempol-mediated activation of latent iron regulatory protein activity prevents symptoms of neurodegenerative disease in IRP2 knockout mice. Proc Natl Acad Sci U S A 105, 12028-12033.

Ginzburg, Y., and Rivella, S. (2011). Beta-thalassemia: a model for elucidating the dynamic regulation of ineffective erythropoiesis and iron metabolism. Blood 118, 4321-4330.

Grosso, R., Fader, C.M., and Colombo, M.I. (2017). Autophagy: a necessary event during erythropoiesis. Blood Rev 31, 300-305.

Haile, D.J., Rouault, T.A., Tang, C.K., Chin, J., Harford, J.B., and Klausner, R.D. (1992). Reciprocal control of RNAbinding and aconitase activity in the regulation of the ironresponsive element binding protein: role of the iron-sulfur cluster. Proc Natl Acad Sci U S A 89, 7536-7540.

Handschin, C., Lin, J., Rhee, J., Peyer, A.K., Chin, S., Wu, P.H., et al. (2005). Nutritional regulation of hepatic heme biosynthesis and porphyria through PGC-1alpha. Cell 122, 505-515.

Ishii, K.A., Fumoto, T., Iwai, K., Takeshita, S., Ito, M., Shimohata, N., et al. (2009). Coordination of PGC-1beta and iron uptake in mitochondrial biogenesis and osteoclast activation. Nat Med 15, 259-266.

Kaneko, H., Kobayashi, E., Yamamoto, M., and Shimizu, R. (2012). N- and C-terminal transactivation domains of GATA1 protein coordinate hematopoietic program. J Biol Chem 287, 21439-21449.

Lin, J., Handschin, C., and Spiegelman, B.M. (2005). Metabolic control through the PGC-1 family of transcription coactivators. Cell Metabol 1, 361-370.

Meyron-Holtz, E.G., Ghosh, M.C., Iwai, K., LaVaute, T., Brazzolotto, X., Berger, U.V., et al. (2004). Genetic ablations of iron regulatory proteins 1 and 2 reveal why iron regulatory protein 2 dominates iron homeostasis. EMBO J 23, 386-395.

Muckenthaler, M.U., Rivella, S., Hentze, M.W., and Galy, B. (2017). A red carpet for iron metabolism. Cell 168, 344-361.

O'Hagan, K.A., Cocchiglia, S., Zhdanov, A.V., Tambuwala, M.M., Cummins, E.P., Monfared, M., et al. (2009). PGC1alpha is coupled to HIF-1alpha-dependent gene expression by increasing mitochondrial oxygen consumption in skeletal muscle cells. Proc Natl Acad Sci U S A 106, 2188-2193.

Pondarre, C., Antiochos, B.B., Campagna, D.R., Clarke, S.L., Greer, E.L., Deck, K.M., et al. (2006). The mitochondrial ATP-binding cassette transporter Abcb7 is essential in mice and participates in cytosolic iron-sulfur cluster biogenesis. Hum Mol Genet 15, 953-964.

Pondarre, C., Campagna, D.R., Antiochos, B., Sikorski, L., Mulhern, H., and Fleming, M.D. (2007). Abcb7, the gene responsible for $\mathrm{X}$-linked sideroblastic anemia with ataxia, is essential for hematopoiesis. Blood 109, 3567-3569.

Rensvold, J.W., Ong, S.E., Jeevananthan, A., Carr, S.A., Mootha, V.K., and Pagliarini, D.J. (2013). Complementary RNA and protein profiling identifies iron as a key regulator of mitochondrial biogenesis. Cell Rep 3, 237-245.

Rouault, T.A. (2015). Mammalian iron-sulphur proteins: novel insights into biogenesis and function. Nat Rev Mol Cell Biol 16, 45-55.

Rouault, T.A. (2016). Mitochondrial iron overload: causes and consequences. Curr Opin Genet Dev 38, 31-37.
Rouault, T.A., and Maio, N. (2017). Biogenesis and functions of mammalian iron-sulfur proteins in the regulation of iron homeostasis and pivotal metabolic pathways. J Biol Chem 292, 12744-12753.

Salahudeen, A.A., Thompson, J.W., Ruiz, J.C., Ma, H.W., Kinch, L.N., Li, Q., et al. (2009). An E3 ligase possessing an iron-responsive hemerythrin domain is a regulator of iron homeostasis. Science 326, 722-726.

Schmitz-Abe, K., Ciesielski, S.J., Schmidt, P.J., Campagna, D.R., Rahimov, F., Schilke, B.A., et al. (2015). Congenital sideroblastic anemia due to mutations in the mitochondrial HSP70 homologue HSPA9. Blood 126, 2734-2738.

Tahara, T., Sun, J., Igarashi, K., and Taketani, S. (2004a). Heme-dependent up-regulation of the alpha-globin gene expression by transcriptional repressor Bach1 in erythroid cells. Biochem Biophys Res Commun 324, 77-85.

Tahara, T., Sun, J., Nakanishi, K., Yamamoto, M., Mori, H., Saito, T., et al. (2004b). Heme positively regulates the expression of beta-globin at the locus control region via the transcriptional factor Bach1 in erythroid cells. J Biol Chem 279, 5480-5487.

Tong, W.H., and Rouault, T.A. (2006). Functions of mitochondrial ISCU and cytosolic ISCU in mammalian iron-sulfur cluster biogenesis and iron homeostasis. Cell Metab 3, 199-210.

Vashisht, A.A., Zumbrennen, K.B., Huang, X., Powers, D.N., Durazo, A., Sun, D., et al. (2009). Control of iron homeostasis by an iron-regulated ubiquitin ligase. Science 326, 718-721.

Ye, H., Jeong, S.Y., Ghosh, M.C., Kovtunovych, G., Silvestri, L., Ortillo, D., et al. (2010). Glutaredoxin 5 deficiency causes sideroblastic anemia by specifically impairing heme biosynthesis and depleting cytosolic iron in human erythroblasts. J Clin Invest 120, 1749-1761.

Zhang, D.L., Ghosh, M.C., and Rouault, T.A. (2014). The physiological functions of iron regulatory proteins in iron homeostasis - an update. Front Pharmacol 5, 124.

Address correspondence to: Stefano Rivella, PhD Division of Hematology Department of Pediatrics

Children's Hospital of Philadelphia University of Pennsylvania 3615 Civic Center Boulevard Philadelphia, PA 19104

USA

E-mail: rivellas@email.chop.edu

Ping La, PhD

Division of Hematology Department of Pediatrics

Children's Hospital of Philadelphia University of Pennsylvania 3615 Civic Center Boulevard Philadelphia, PA 19104

USA

E-mail: laping3@gmail.com

Received for publication September 23, 2019; received in revised form February 20, 2020; accepted February 21, 2020. 